\title{
Review of the Use of Bioindication Method for Appraisal of the State of Urban Soils in Two Areas of Saint Petersburg, Russia
}

\author{
K. A. Gyekye \\ Department of Geography and Resource Development, P. O. Box LG 59, Legon, Ghana
}

\begin{abstract}
The paper examines the use of culture of Paramecium caudatum (infusoria) as an indicator to determine soil toxicity of urban soils within the areas of Vsileostrovsky and Elagin Ostrov in St Petersburg, Russia. The results of the study indicated that urban soils of Vasileostrovsky were toxic; index of soil toxicity $(\mathrm{T})$ ranged within the limits of $0.27-0.77$. Results of tested soils from Elagin Ostrov showed a significant negative index of soil toxicity, which ranged from -0.86 to -1.26 , specified that soils were not toxic. Comparing results from this study to the study conducted on ecological zoning of Vasileostrovsky, on the basis of green plants reaction to complex pollutants, showed wide disparities between the two bioindication approaches, which was attributed to specific biological characteristics of species tolerance (test organisms) and their levels of sensitivity to complex ecological factors. In view of the growing need to conserve soils in urban areas the investigation, which used paramecium caudatum as bioindicator to detect soil toxicity, will promote monitoring of state of soils in developing cities.
\end{abstract}

\section{Introduction}

It is generally acknowledged that urbanization leads to transformation of natural landscapes and dramatic changes in the state of natural resources such as water, soils, vegetation, atmospheric air and climatic conditions. In place of natural soils, specific soils, called urban soils (urbic), are formed (Kuprianov, 1977; 0simov \& Perelman, 1995; Dobrovolsky, 1997). Located in a semi-marshy environment, the development of St Petersburg has resulted in substantial changes of its natural resources. A change in the state of natural resources of the urban environment quite often creates critical ecological situation the state of which can be assessed and monitored by the use of physical, chemical and biological methods.

The ecological status of St Petersburg has been basically associated with automobile and industrial emissions (Baeva, 1995). Several studies carried out on the ecological state of St Petersburg on such components such as soils, air pollution, heavy metals, plants and macrobenthic invertebrates by physical, chemical and biological methods revealed not only serious ecological threats but also wide disparities in research results (Andreiva \& Baeva, 1995, 1996, 1998, 2000, 2002; Terekhina, 1998; Ufimtseva \& Terekhina, 2000).

The disparities of research results obtained on the ecological state of various sections of urban areas within St Petersburg, for example, Vasileostrovsky (Fig. 2) create ambiguity about the ecological state of the city and, thus, makes it difficult to take appropriate decisions on monitoring of urban natural resources. Such circumstances specified the need to review the ecological state of sections of St Petersburg by using bioindication method (in this study, by using Paramecium caudatum) as means of verifying such disparities. The use of Paramecium caudatum (chemotaxis infusoria) as a test-object has the potential to determine the presence of toxicity of a substance in the environment (Soldo \& Merlin, 1972; Berk et al., 1978, Belichinskaya, 2000).

It is considered that one of the reliable methods for assessing the ecological state of the urban environment is the use of bioindicators. Bioindicators react to data and complex information on environmental conditions and, thus, provide a fair assessment on the ecological state of the environment. It also forms the basis of adopting preventive measures and for selecting more reliable methods for further research into the natural components of the urban environment (Rolya, 1990; Ufimtseva \& Terekhin, 1996). However, it is far from being always true that conclusions made on the basis of bioindication results reflect the true environmental situation on the ground (Alekseev et al., 2002; Alimova et al., 1999).

The widely use of bioindicators to assess the complex state of the natural environment and for determining specific problems related to monitoring of the environment justify the need to adopt

West African Journal of Applied Ecology - Volume 13 
bioindication methods for this research (Seliverstov, 1994; Pozharov et al., 1998; Ufimtseva \& Terekhin, 2000; Chibrik, 2001). Such approaches have the greatest value in their application to the studying of larger cities (for example, Saint Petersburg with a population of about 4 million), where there are environmental degradation caused by human developments such as industrial activities and transport.

As part of the review strategy and to help compare results, two quite distinct areas within St Petersburg were selected for the study; these were Vasileostrovsky and Elagin Ostrov. Apparently, the two chosen study areas differ from each other in terms of the nature of ecological characteristics and the level of human impacts on the environment. Located near to the central part of St Petersburg city, Vasileostrovsky faces increasing ecological threats due to its high population and the attendant negative human impacts as compare to Elaging Ostrov, situated about $3 \mathrm{~km}$ from the center of St Petersburg, and functions as a recreational zone. Generally, the environmental problems, as stated above, require detailed and comprehensive review of the ecological status of St. Petersburg to asses the extent of negative human impacts, and to provide the requisite data to enable the monitoring of anthropogenic impacts over time.

The paper reviews the use of bioindication method for the appraisal of the state of urban soils and to provide a basis for sustainable urban soils management. The specific objectives of the study were to (i) examine the presence and level of soil toxicity in urban soils by using Paramecium caudatum (infusoria) as an indicator, (ii) compare the results of the study to the results of previous findings by other researchers using bioindication methods to assess soil toxicity within the selected study area, and (iii) provide a better understanding of the disparity nature of the ecological zoning of Vasileostrovsky and to come out with appropriate recommendation for sustainable management of urban soils.

\section{Materials and methods}

Study area

The study covered two areas, namely Vasileostrovsky and Elagin Ostrov, all located within St Petersburg (Fig.1 and 2). Even though the two study areas are fairly close to each other, they are quiet distinct from each other in terms of land use pattern: Vasileostrovsky is an administrative unit of St Petersburg and is characterized by settlements, commercial and light industrial activities. Elagin Ostrov, sited about $3.5 \mathrm{~km}$ north of Vasileostrovsky, serves exclusively as a recreational zone. The differences in land use pattern and the availability of data made it convenient to select Elagin Ostrov and Vasileostrovsky as model territories to carry out a comparative study on the scale of human influence on these two apparently different environments.

West African Journal of Applied Ecology - Volume 13 


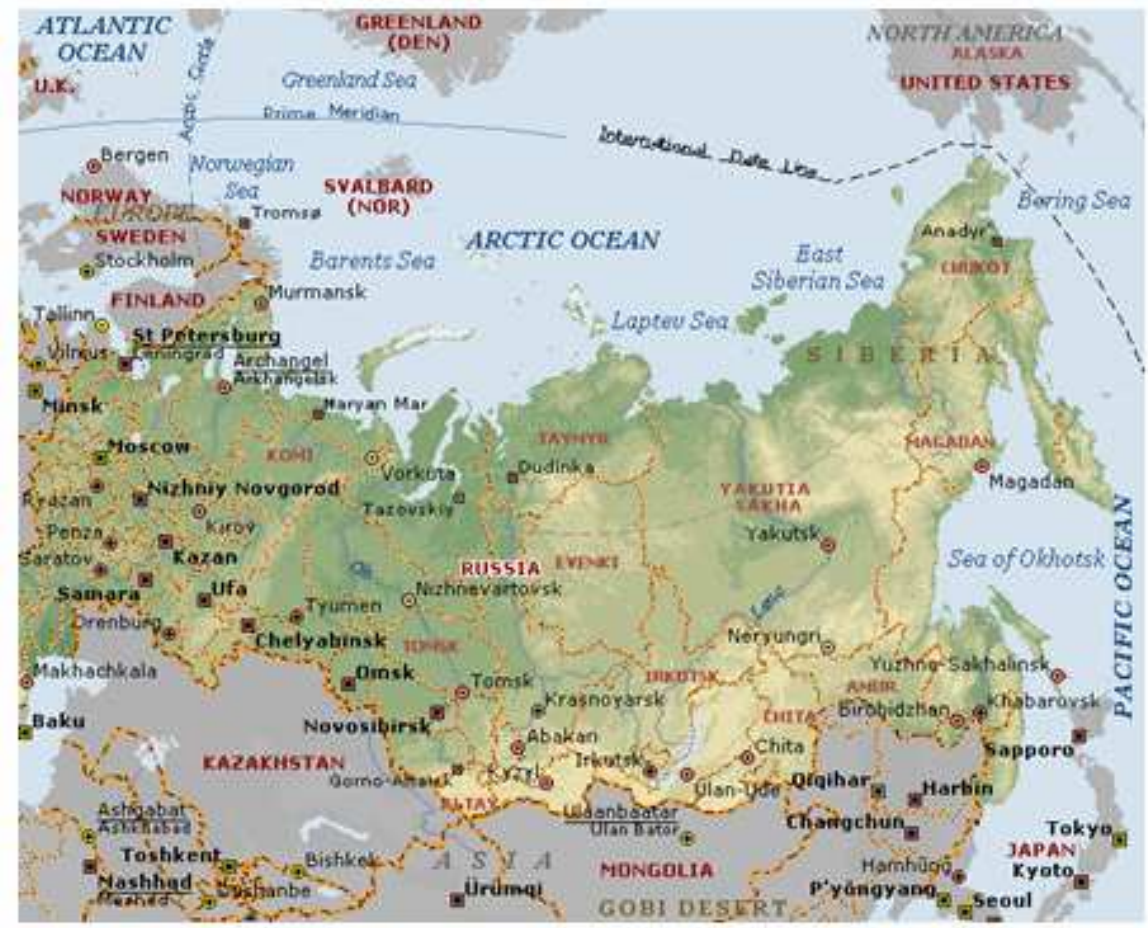

Fig. 1. Map of Russia Showing location of St. Petersburg

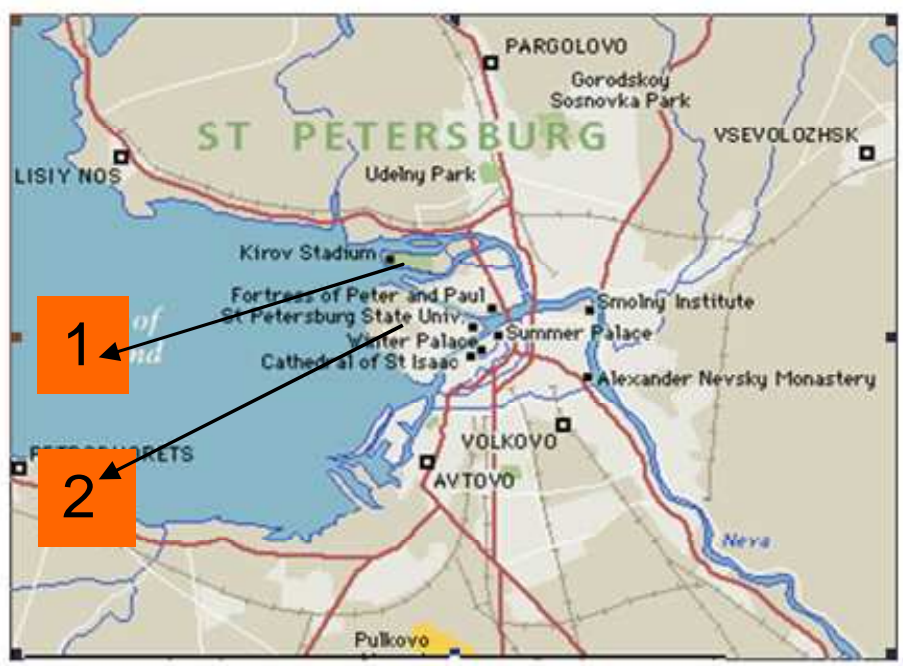

Fig 2. Map of St. Petersburg showing the two study areas of Vasileostrovsky and Elagin Ostrov

The methodology involved an evaluation of literature on selected bioindicators, reviewed of data from previous researchers on physical and chemical characteristics of various components of the urban environ-ment, which forms the baseline information for the study. The field work involved examining the characteristics of natural and anthropogenic factors that are likely to influence the character of urban environment and research results. These included visual assessment of selected study sites to determine the nature of land use, relief, drainage characteristics and vegetative cover. Information on the above was used to specify sampling sites.

West African Journal of Applied Ecology - Volume 13 


\section{Soil sampling sites}

Soil samples from Vasileostrovsky were obtained from road sides and some distance away from the roads, industrial fringes, parks and gardens, and settlement areas. Such carefully planned selection was to help determine the pattern and the character of an indicator's reaction to different points of land use. Sampled points included those points which have been previously investigated by other researchers such as Terekhina (1998) and Ufimseva \& Terekhina (2000) using express methods of plant-indication. Within Elagin, soil samples were taken from different environment -road edges, areas burdened with recreational activities, slopes, footpaths and flat plains.

Total number of sampled points was 22. Eighteen samples were taken from Vasileostrovsky and only four from Elagin Ostrov. The difference in numbers of sampled points was due to not only the difference in size between the two study areas but also that samples taken from Elgin Ostrov were used as a comparative measure with samples taken from Vasileostrovsky. Sampled soils were taken with the aid of stainless tool known as sheath Dutch anger. Sampling depth was $0-20 \mathrm{~cm}$ which is considered as the active biological zone and acts as barrier to atmospheric pollutants on deeper soils (Gaborvskaya et al., 2001). Samples were kept in labeled polyethylene bags and stored under laboratory conditions.

To determine the presence and level of soil toxicity, bioindication method based on chemotaxis reaction of Paramecium caudatum (infusoria) was used (Soldo \& Merlin, 1972; Berk et al., 1985; Gills \& Roger, 2002). For assessing soil toxicity, 5\% solution of polyvinyl alcohol (PVA) was prepared by boiling $0.05 \mathrm{~g}$ PVA in $0.95 \mathrm{ml}$ distilled water. A visible boundary between two separate zones in a photometric ditch was created by adding $1.32 \mathrm{ml}$ of $10 \%$ solution of eosin indicator to the prepared solution of PVA. To the photometric ditch was added $2.0 \mathrm{ml}$ of extracted soil solution on the layer of the mixed PVA and eosin, and pure culture of infusoria.

The boundary section between the two liquid environments does not interfere with the free movement of infusoria. Simultaneously, control experiment was conducted in Lozina Lozinsky (LL) medium by using copper salt solution. Both test soils and control test, in separate photometric ditches, were placed in a Biotester. Time for an exposition was $15 \mathrm{~min}$. During this period infusoria were distributed in the following ways: if the tested soil solution is toxic infusoria remain at the bottom of the ditch. On the other hand, if tested soil solution is clean infusoria move towards the surface (Soldo \& Merlin, 1972). Control value for each sampled soil was measured and, in each case, a value of 38.86 was recorded with the only exception of sample point 30 , in which case, and after two consecutive measurements, a control value of 27.40 was recorded. There was no immediate account for arriving at this figure, therefore, investigation into the physical and chemical properties of the sampled soil, and operation of the biotester was regarded necessary.

At the end of the experiment, the index of toxicity as expressed by Berk et al., (1985) was calculated under the formula:

$$
T=(\text { Icontr }- \text { Iexp }): \text { Icont } .
$$

where: $T$ refers to index of toxicity, Icontr - Biotester readings for control test; Iexp - Biotester readings for sampled soil test;

\section{Results and discussion}

Results of tested soils from Elagin Ostrov, based on an estimation of reaction of infusoria indicated that sampled soils were not toxic; this is indicated by the significant negative index of soil toxicity, which ranged from -0.86 to -1.26 , as shown in Table 1 . In Vasileostrovsky, the degree of soil toxicity varied significantly from one section of the city to the other. Results indicated that soils from the western part of Vasileostrovsky (considered to be the youngest area in terms of development) were not toxic, registering index of soil toxicity of less than zero $(\mathrm{T}<0:-0.53,-0.76,-0.21)$. The degree of soil toxicity for the rest of Vasileostrovsky varied within the range of -0.21 to 0.77 . Such a wide variation in toxicity values indicated the heterogeneous nature of urban soils. The most toxic soils were found in the southern section West African Journal of Applied Ecology - Volume 13 
of central Vasileostrovsky Island and in the eastern part; within these areas index of soil toxicity ranged from 0.50 to 0.81 , which corresponds to the range of toxic and strongly toxic, respectfully (Table 2).

TABLE 1

Biotest results showing index of soil toxicity at different points of Elagin Ostrov by method of culture Paramecium caudatum (infusoria)

$\begin{array}{cccc}\text { Sampling points } & \begin{array}{c}\text { Biotester readings for } \\ \text { control test }\end{array} & \begin{array}{c}\text { Biotester readings for } \\ \text { sampled soil test (I exp.) }\end{array} & \text { Index of soil toxicity (T) } \\ 46 & 38.86 & 72.70 & -0.86 \\ 51 & 38.86 & 88.50 & -1.28 \\ 61 & 38.86 & 71.69 & -0.84 \\ 69 & 38.86 & 98.72 & -1.54\end{array}$

$T<0$ - non-toxic; $0<T<0.25$ - weakly toxic; $0.25<T<0.5$ - moderately toxic; $0.5<T<0.75$ - toxic; $0.75<T<1.0-$ strongly toxic. (Source: Soldo \& Merlin, 1972; Berk et al., 1985)

TABLE 2

Index of soil toxicity at different points in Vasileostrovsky by method of culture Paramecium caudatum (infusoria)

Sampling points

2
38.86
7
38.86
12
13
14
15
16
17
18
20
21
22
25
26
27
30

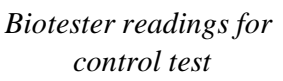

38.86

68.30

38.86

21.42

38.86

38.86

38.86

38.86

38.86

38.86

38.86

38.86

38.86

38.86

38.86

38.86

38.86

27.40
Biotester readings for

sampled soil test (I exp.)
Index of toxicity $(T)$

$-0.53$

$-0.21$

$-0.86$

1.02

$-0.81$

0.50

0.70

0.63

0.51

0.73

0.64

$-2.22$

0.13

$-5.71$

0.05

0.77

$\mathrm{T}<0$ - non-toxic; $0<\mathrm{T}<0.25$ - weakly toxic; $0.25<\mathrm{T}<0.5$ - moderately toxic; $0.5<\mathrm{T}<0.75$ - toxic; $0.75<\mathrm{T}<1.0$ - strongly toxic (Source: Soldo \& Merlin, 1972; Berk et al., 1985)

Many factors have been linked to such high toxic levels such as high density of vehicular movements, industrial activities that are located closely to these study points, and the presence of heavy metals. For example, according to Andreiva et al. (1998), total concentration of heavy metals (TcHM) in soils made up of $\mathrm{Pb}, \mathrm{Cu}, \mathrm{Mg}$ and $\mathrm{Zn}$ in most part of central Vasileostrovsky ranged within TcHM.32-128, which is measured as dangerous, whilst in the eastern part, total concentration of $\mathrm{Pb}, \mathrm{Cu}, \mathrm{Mg}$ and $\mathrm{Zn}$ was found to be in the range of TcHM 128-132, and it is considered as extremely dangerous by Russia standard. Such high concentra-tions of heavy metals above regional permissible levels in soils $(\mathrm{TcHM}<32)$ frequently inhibit the normal functioning of soil ecosystem and have lethal effects on biological activities (Dobrovolsky, 1997; Feodorov, 2002). 
Similar studies confirmed wide variations in physico-chemical parameters. For example, the $p \mathrm{H}$ value for Vasileostrovsky ranged within 4.7-8.1, whilst $\mathrm{Ca}^{2+}$ and $\mathrm{Mg}^{2+}$ ranged within 2.4-28.1 m.eq/100 $\mathrm{g}$ of soil and for total basic cation exchange, $12-48 \mathrm{meq} / 100 \mathrm{~g}$ of soil (Movchan et al., 1997). Various investigations have also confirmed significant correlation between $p \mathrm{H}$ and parameters of biological activities of soils (Dobrovolsky \& Nikitin, 1986; Babjeva \& Zenov, 1989; Movchan et al., 1997). Such significant variations in physico-chemical characteristics of the study area might have accounted for the wide differences in infusorian's reaction to soil pollutants and, for that matter, the variations in the levels of soil toxicity. However, the study did not reveal any direct relationship between site-specific conditions such as the level of $p \mathrm{H}$, organic contents, $\mathrm{Ca}^{2+}$ and $\mathrm{Mg}^{2}$, etc. and levels of soil toxicity. This explains the fact that the level of soil toxicity may depend on complex environmental factors and not just on few selected parameters.

Generally, low index of soil toxicity (0-0.25) were observed, mostly in settlement areas and areas outside high traffic density zones. There was no immediate explanation to account for such pattern of distribution. Even though high values of toxic soils were observed mainly in the south-western part, as shown in Fig. 3, low levels of soil toxicity were also noted. Thus, it was observed that areas with high soil toxicity level, with an average value of 0.75 (this is generally found between the south-western and eastern parts), also recorded low values of toxicity ranging from 0 to 0.50 . Similarly, in the north-western part, both non toxic and moderately toxic soils were recorded. Such anomalies in the distribution pattern might be due to site specific conditions, which call for further investigation into such anomalies.

It can be said from the results obtained that the influence of territorial heterogeneity of urban soils on the level of soil toxicity was clearly noted, toxic soils varied from non-toxic, weakly toxic to moderately toxic soils (Fig. 3). Thus, a careful assessment of urban soils based on method of chemotaxis infusoria revealed territorial differences in terms of the quality components of urban environment from the point of view of essential conditions necessary for hetero-trophe nature of living organisms. Again, it is interesting to note the reaction of infusoria from the various tested soils taken from Vasileostrovsky. Within this territory, the reaction of infusoria indicated that tested soils were toxic, particularly in the south-eastern portions of the central part and to the eastern part of Vasiloestrovsky, whilst the western and a greater part of eastern Vasileostrovsky and Elagin revealed that soils were not toxic. Such wide variations in biotest results could be explained as specific biological characteristics of species tolerance (test organisms) and levels of sensitivity to different environmental conditions. Com-paratively, results obtained for all analysed soils sampled from different section of Elagin recoded negative values and showed insignificant variation.

West African Journal of Applied Ecology - Volume 13 


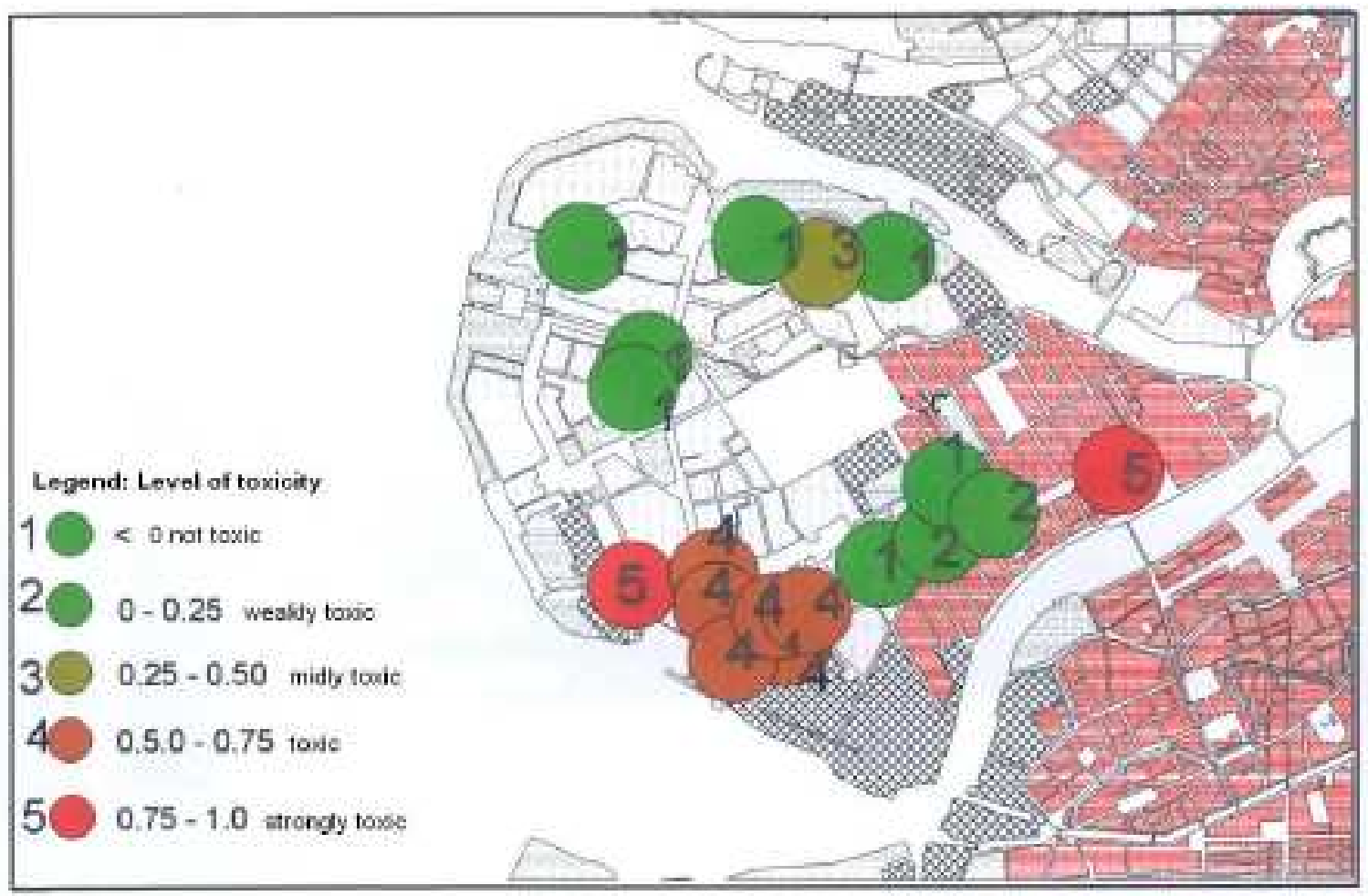

Fig.3. Map of Vasileostrovsky showing Index of soil toxicity

On the whole, it can be argued that soils in Elagin Island were more favourable for living organisms than soils in Vasileostrovsky area; the former is most likely due to insigni-ficant human impacts on the environment and effects of landscape gardening services carried out in Elagin to improve soil conditions (Mets et al., 1989). A compara-tive analysis of the results of this study on biotest submitted above with information on ecological zoning of Vasileostrovsky (Fig. 4), which was carried out on the basis of green plants (bioindication) reaction to complex environmental conditions (Terekhina, 1998), showed wide disparities between the two results. It was observed, for example, that zones considered to be of highest ecological risk according to Terekhina (1998) (which included sites investigated by this study) were classified as toxic according to Paramecium reaction to soils from these zones. However, in other areas zoned as unsatisfactory, soil toxicity ranged from non toxic, weak toxic $(0-25)$ to strong toxic $(0.75-1.0)$. Besides, tested urban soils, taken from a greater part of areas classified as ecologically risk zones, were found to be non-toxic by the results of Paramecium caudatum.

West African Journal of Applied Ecology - Volume 13 


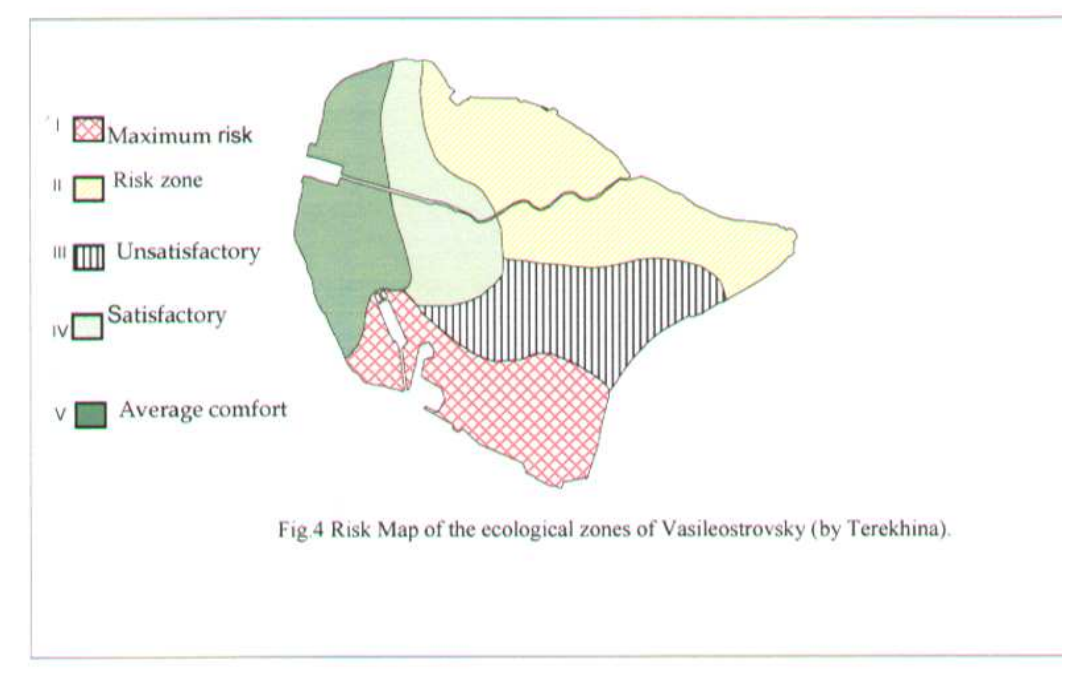

\section{Conclusion}

The study demonstrated that Paramecium caudatum was capable of registering the presence of toxic pollutants in soils. Soils tested from Elagin were classified as not toxic as compared to Vasileostrovsky, where soil toxicity ranged from non-toxic to strong toxic. The results revealed influence of heterogeneous nature of urban environment on the level of soils toxicity and this cor-relates with land use pattern. Paramecium caudatum was found to be, generally, more sensitive to soils that are highly under anthropogenic pressure which suggested the need to adopt $P$. caudatum for monitoring urban environment which is always under anthropogenic pressure. Preferably, two or more indicators should be used to assess the presence of toxic substances in soils. This would help one to draw a comparative measure between different species reaction to pollutants in urban soils.

On the basis of this research results, it can be said that the ecological zoning of Vasileostrovsky on the results of bioindication reflects not so much on technogenic impacts (in the first place, chemical pollution as it is most often considered), but are as a result of complex ecological factors. To determine the trend of soil toxicity, it is recommended that the study should be carried out on regular basis and its scope extended. This would provide more detail information not only on the level of toxicity in soils but also to determine the character of indicators reaction to different environmental conditions. Appropriate and efficient land use mechanism is recommended to reduce the level of anthropogenic emissions of pollutants into urban environment.

\section{Acknowledgement}

The author wishes to thank Prof. V. M Movchan, Head of Department, Geography and Resource Use, Saint Petersburg State University, Russia. His gratitude also goes to Dr Gorbovskaya Alla Danilovna, Saint Petersburg State University, Russia, and Nadieshda Ambrarovna, Head of ECOLAB, Department of Geography and Resource Use, Saint Petersburg State University, for their assistance in the analysis and identification of specimens.

\section{References}

Aleekseev D. K. and Galisova V. V. (2002). Assessing Ecological state of deep deposits of the Klotsova Gulf of the Baren Sea Quest. appl. Ecol. SPB, RGMU: 65-70.

Alimova A. F., Dmitriev V. V. and Florinskaja T. A. (1999). An Integrated Assessment of the Ecological Condition and Quality of the Environment of Urban Territories. NSC, St Petersburg. 253 pp.

West African Journal of Applied Ecology - Volume 13 
Anderiva B. G., Baeva A. C. and Bakova N. V. (1995). Protection of the Environment, Resource Use and Ecological Safety of St Petersburg. St Petersburg.

Anderiva B. G., Baeva A. C. and Bakova N. V. (1996). Protection of the Environment, Resource Use and Ecological Safety of St Petersburg. St Petersburg.

Anderiva B. G., Baeva A. C. and Bakova N. V. (1998). Protection of the Environment, Resource Use and Ecological Safety of St Petersburg. St Petersburg.

Andreiva B. G., Baeva A. C. and Bakova N. V. (2000). Protection of the Environment, Resource Use and Ecological Safety of St Petersburg. St Petersburg.

Anderiva B. G., Baeva A. C. and Bakova N. V. (2002). Protection of the Environment, Resource Use and Ecological Safety of St Petersburg. St Petersburg.

Babjeva I. P. and Zenov G. M. (1989). Biology of Soils. Moscow State University. 336 pp.

Belichinskaya L. I. (2000). Bioindication of industrial toxicants on wood plants. Forestry College, Voronezh. p. 93.

Berk S. G., Gunderson J. N. and Derk L. A. (1985). Effects of cadmium and cupper on chemotaxis of marine and freshwater ciliates. Bull. Envir. Center Technol. 34: 897-903.

Chibrik T. S. (ed.) (2001). Plants and the industrial environment. Eleventh (XI) International Symposium on bioindicators " Contemporary problems of Dioindication and biomonitoring ». Syktyvkar, September, 1-15, 2001. 140 pp.

Dobrovolsky G. V. and Nikitin E. D (1986). Ecological functions of soils: Test book for universities specializing in agrochemistry and soil science. M. Publishing House, Moscow State University. p. 136.

Dobrovolsky G. V. (1997). Soils, Cities, Ecology. Science Publishers, Moscow. 332 pp.

Feodorov A. C. (2002). Laws of formation of soil stability against anthropogenic influences (for example, pollution by heavy metals and an agricultural production). (PhD Thesis.) Saint Petersburg. p. 44.

Gills Rick and Roger J. (2002). Molecular and Cytochemical Characterization of the Holospora infection in Paramecium caudatum. Department of Biology, University of Wisconsin, La Crosse.

Gorbovskaya A. D., Movchan V. N. and Gersh B. N. (2001). The ecological condition of a soil cover of Kostomuksha and adjoining territories; Bulletin of St. Petersburg State University. Serial \#. 7, 1 st edition (\#7). pp. 135-140.

Kasimov N. S and Perelman A. I. (1995). Ecogeochemistry of city landscapes. Moscow State University. p. 333.

Kuprianov V. V. (1997). Hydrological aspects of urban environment Leningrad. Hydro-publication. p. 184.

Mets A. R., Tsvetkova S. D. and Melinuchuk I. A. (1989). Characteristics of morphological and agrochemical properties of soils of Kirovsky and Pabedi Parks of the Seaside. Forestry, Wood Cultures and Soil Science. University Collection, Leningrad. pp. 73-78.

Movchan V. N., Gorbovskaya A. D. and Smiths Y. V. (1997). The ecological state of urban soils. A case study of Saint Petersburg. In Ecological monitoring of model territories of Northwest Russia (V. N. Movchan, ed.), pp, 35-37. St Petersburg State University.

Pozharov A. V., Paputskaja N. I. and 3axapoB I. S. (1998). Express toxicological test by the use of chemotaxis Paramecium caudatum (Infusoria) for biotesting. St Petersburg.

Pronin N. B. (2000). Ecological Stresses: Reasons, Classification, Testing and Physicobiochemical Mechanisms. Publishing House MSHA, Moscow. 312 pp.

Rolya J. (1990). Use of bioindication method for an estimation of residual amounts of herbicides in soils and their overall phytoindication. Moscow. 313 pp.

Seliverstov J. U. P. (1994). Concepts and problems of ecological and geographical assessment of the state of the natural environment (P. P Agapova and J. U. P Seliverstov, ed.), pp. 5-17. St Petersburg.

Soldo A. T. and Merlin E. J. (1972). The cultivation of symbiotic- free marine ciliates in arsenic medium. J. Protocol. 19: 519.

Tarhanen S. (1996). Effect of industrial emissions on membrane permeability of epiphytic lichens in northern Finland and the Kola Peninsula industrial areas. Wat. Air Soil Pollut. 88 (1-2): 189-201.

Terekhina N. V. (1998). Complex criteria of using plant indication to assess the ecological state of an urban environment. $(\mathrm{PhD}$ Thesis.) Geogra-phical Sciences, Saint Petersburg. p. 20.

Ufimtseva M. D. and Terekhina N. V. (1996). Plant indication research in monitoring urban environment (for example, Saint Petersburg). Materials from the International Congress on « Ecological Initiative - 96 », Voronezh. p. 22.

Ufimtseva M. D. and Terekhina. N. V. (2000). Express (quick) method by plant indication for an estimation of the ecological state of the environment Publishing House of St Petersburg State Univer-sity. p. 32.

West African Journal of Applied Ecology - Volume 13 